This is an accepted manuscript of an article published by Taylor \& Francis in Complex Variables and Elliptic Equations on 20 Jan 2021, available online:

https://doi.org/10.1080/17476933.2020.1868447

\title{
The Swallowtail integral in the highly oscillatory region III
}

\author{
Chelo Ferreira ${ }^{1}$, José L. López ${ }^{2}$ and Ester Pérez Sinusía ${ }^{1}$ \\ ${ }^{1}$ Dpto. de Matemática Aplicada, IUMA, Universidad de Zaragoza, Zaragoza \\ e-mail: cferrei@unizar.es, ester.perez@unizar.es \\ ${ }^{2}$ Dpto. de Ingeniería Matemática e Informática, Universidad Pública de Navarra and INAMAT, Pamplona \\ e-mail: jl.lopez@unavarra.es
}

\begin{abstract}
We consider the swallowtail integral $\Psi(x, y, z):=\int_{-\infty}^{\infty} e^{i\left(t^{5}+x t^{3}+y t^{2}+z t\right)} d t$ for large values of $|z|$ and bounded values of $|x|$ and $|y|$. The integrand of the swallowtail integral oscillates wildly in this region and the asymptotic analysis is subtle. The standard saddle point method is complicated and then we use the modified saddle point method introduced in [López et al., 2009]. The analysis is more straightforward with this method and it is possible to derive complete asymptotic expansions of $\Psi(x, y, z)$ for large $|z|$ and fixed $x$ and $y$. The asymptotic analysis requires the study of three different regions for $\arg z$ separated by three Stokes lines in the sector $-\pi<\arg z \leq \pi$. The asymptotic approximation is a certain combination of two asymptotic series whose terms are elementary functions of $x, y$ and $z$. They are given in terms of an asymptotic sequence of the order $\mathcal{O}\left(z^{-n / 12}\right)$ when $|z| \rightarrow \infty$, and it is multiplied by an exponential factor that behaves differently in the three mentioned sectors. The accuracy and the asymptotic character of the approximations is illustrated with some numerical experiments.
\end{abstract}

2010 AMS Mathematics Subject Classification: 33E20; 41A60.

Keywords \& Phrases: Swallowtail integral. Asymptotic expansions. Modified saddle point method. 


\section{Introduction}

The mathematical models of many short wavelength phenomena, specially wave propagation and optical diffraction, contain, as a basic ingredient, oscillatory integrals with several nearly coincident stationary phase or saddle points. The uniform approximation of those integrals can be expressed in terms of certain canonical integrals and their derivatives [2], [15]. The importance of these canonical diffraction integrals is stressed in [13] by means of the following sentence: The role played by these canonical diffraction integrals in the analysis of caustic wave fields is analogous to that played by complex exponentials in plane wave theory.

Apart from their mathematical importance in the uniform asymptotic approximation of oscillatory integrals [11], the canonical diffraction integrals have physical applications in the description of surface gravity waves [10], [16], bifurcation sets, optics, quantum mechanics and acoustics (see [1, Sec. 36.14] and references there in).

In [1, Chap. 36] we can find a large amount of information about this integrals. First of all, they are classified according to the number of free independent parameters that describe the type of singularities arising in catastrophe theory, that also corresponds to the number of saddle points of the integral. The simplest integral with only one free parameter, that corresponds to the fold catastrophe, involves two coalescing stationary points: the well-known integral representation of the Airy function. The second one, depending on two free parameters corresponds to the cusp catastrophe and involves three coalescing stationary points. The third one, depending on three free parameters corresponds to the swallowtail catastrophe and involves four coalescing stationary points. The canonical form of the oscillatory integral describing the swallowtail diffraction catastrophe is given by the swallowtail catastrophe integral [1, eq. 36.2.4]:

$$
\Psi(x, y, z):=\int_{-\infty}^{\infty} e^{i\left(t^{5}+x t^{3}+y t^{2}+z t\right)} d t .
$$

Apart from the classification of this family of integrals, in [1, Chap. 36] we can find many properties such as symmetries, illustrative pictures, bifurcation sets, scaling relations, zeros, convergent series expansions, differential equations and leading-order asymptotic approximations among others. But we cannot find complete asymptotic expansions.

The first three canonical integrals: Airy function, Pearcy integral and swallowtail integral are the most important ones in applications. The first one is well-known and has been deeply investigated in the literature. The second one has been considered in recent works [7, 8] and other more classical works [5, 13, 14]. In this paper we focus our attention in the third one. In [1, eq. 36.8.1] we can find the convergent expansion:

$$
\Psi(x, y, z)=\frac{2}{5} \sum_{n=0}^{\infty} i^{n} \cos \left(\frac{\pi(4 n-1)}{10}\right) \Gamma\left(\frac{n+1}{5}\right) a_{n}(x, y, z),
$$

where $a_{0}(x, y, z)=1$ and, for $n=0,1,2, \ldots$,

$$
a_{n+1}(x, y, z)=\frac{i}{n+1} \sum_{p=0}^{\min (n, 2)}(p+1) \hat{x}_{p+1} a_{n-p}(x, y, z),
$$


with $\hat{x}_{1}=z, \hat{x}_{2}=y, \hat{x}_{3}=x$. The convergence speed of this expansion is rather slow for moderate or large values of the variables. In [1, eq. 36.11.2] we can find the leading order approximation of $\Psi(x, y, z)$ in terms of elementary functions, but it is valid only when the stationary points of the phase function are real and distinct. In [6] we can find an asymptotic approximation of $\Psi(x, y, z)$ in terms of Pearcey integrals, valid for large negative $x$ with $y$ real, and that remains valid when $x, y, z$ are near the cusp of the caustic.

In [3] we investigated the asymptotic behavior of $\Psi(x, y, z)$ for large $|x|$ and fixed $y$ and $z$. In [4] we investigated the asymptotic behavior of $\Psi(x, y, z)$ for large $|y|$ and fixed $x$ and $z$. In this work we derive new asymptotic expansions that produce satisfactory approximations of $\Psi(x, y, z)$ for large $|z|$ and moderate values of $x$ and $y$. The analysis here is different from the analysis in [3] and [4], as the location of the saddle points and the analysis of the steepest descent paths are different. As a consequence, the complex $z$-plane is divided in asymptotic regions different from those found in [3] for the $x$-plane or in [4] for the $y$-plane, and the Stokes lines are also different.

In the following section, we analyze the saddle point features of the swallowtail integral for large $|z|$ and fixed $x$ and $y$. Section 2 contains some preliminaries necessary for the asymptotic analysis. In Section 3 we analyze the saddle points and steepest descent paths of the quadratic part of the phase function. In Section 4 we analyze the deformation of the integration path. In Section 5 we compute the integrals over the asymptotically relevant saddle points. Section 6 contains a summary of the discussion and some numerical experiments. Throughout all the paper we use the notation $\theta:=\arg z$ and use the principal argument $\arg w \in(-\pi, \pi]$ for any complex number $w$ and the notation $w^{*}$ for the complex conjugate of $w$.

\section{Preliminaries}

The integral (1) exists only for $0<\arg y<\pi$ and real $x$, or for real $x, y$ and $z$. After splitting the integration interval $(-\infty, \infty)$ at $t=0$ and rotating the path $(-\infty, 0)$ an angle $-\pi / 10$, and the path $(0, \infty)$ and angle $\pi / 10$, the swallowtail integral may be written in the form

$$
\Psi(x, y, z):=\int_{L} e^{i\left(u^{5}+x u^{3}+y u^{2}+z u\right)} d u
$$

where $L:=\left(\infty e^{9 i \pi / 10}, 0\right] \cup\left[0, \infty e^{i \pi / 10}\right)$ is the path depicted in Figure 1. This last integral is absolutely convergent for all complex values of $x, y$ and $z$. Therefore, the right hand side of (4) is an analytic representation of the analytic continuation of the swallowtail integral $\Psi(x, y, z)$ to all complex values of $x, y$ and $z$. 


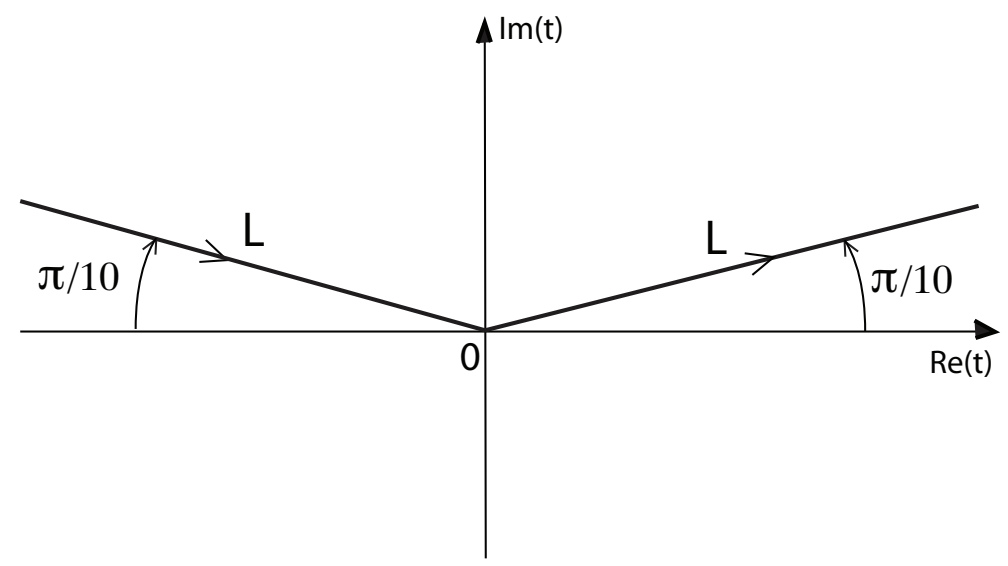

Figure 1: The path $L$ is the union of two half-straights joined at the origin $t=0$ and form an angle of $\mp \pi / 10$ radians with the negative and positive real axes respectively.

\section{Saddle points and steepest descent paths of $\Psi(x, y, z)$}

After the change of variable $u=t\left(\frac{|z|}{5}\right)^{1 / 4}$ in the integral (4) we find that

$$
\Psi(x, y, z)=\left(\frac{|z|}{5}\right)^{1 / 4} \int_{L} e^{\left(\frac{|z|^{5}}{5}\right)^{1 / 4} f(t)+i x\left(\frac{|z|}{5}\right)^{3 / 4} t^{3}+i y\left(\frac{|z|}{5}\right)^{1 / 2} t^{2}} d t
$$

where we have defined the phase function $f(t):=i e^{i \theta} t+\frac{i}{5} t^{5}$. This phase function has four saddle points: $t_{k}:=e^{i(\theta+(2 k-1) \pi) / 4}, k=1,2,3,4$; or $t_{1}=e^{i(\theta+\pi) / 4}, t_{2}=i t_{1}, t_{3}=i t_{2}, t_{4}=i t_{3}$. From the steepest descent method [17, Chap. 2], or its simplified modification [9], we know that the asymptotically relevant saddle points are those ones for which the integration path $L$ in (5) can be deformed into a steepest descent path (or union of steepest descent paths) that contains the relevant saddle points. We will show below that only the saddle points $t_{1}$ and $t_{2}$ are relevant in the asymptotic analysis.

The analytic expression of the steepest descent paths of $f(t)$ at the saddle points is not straightforward. On the other hand, we know from [9] that the asymptotic analysis of the integral (5) does not require the computation of the steepest descent paths of $f(t)$ at the saddle points $t_{k}$, but the steepest descent paths of the "main part" of $f(t)$ at $t_{k}$, that may always be computed in a straightforward manner, as they are nothing but straight lines [9].

At the four saddle points $t_{k}, k=1,2,3,4$, we have that the first non-vanishing derivative of $f(t)$ is $f^{\prime \prime}(t)$. Then, following the notation of [9], at every saddle point $t_{k}$, we denote by $\phi_{k}$ the phase of $f^{\prime \prime}\left(t_{k}\right)$ and by $f_{2}(t)$ the Taylor polynomial of degree 2 of $f(t)$ at the saddle point: $f_{2}(t):=f\left(t_{k}\right)+f^{\prime \prime}\left(t_{k}\right)\left(t-t_{k}\right)^{2} / 2$. At every steepest descent straight, the "main part" of $f(t)$ is just $f_{2}(t)$ and, at each saddle point $t_{k}, k=1,2,3,4$, we have that the steepest descent paths of $f_{2}(t)$ are the following straights trough the saddle points [9]:

$$
\Gamma_{k}=\left\{t_{k}+r e^{i \theta_{k}} ; \quad \theta_{k}=\frac{\pi-\phi_{k}}{2} ;-\infty<r<\infty\right\} .
$$




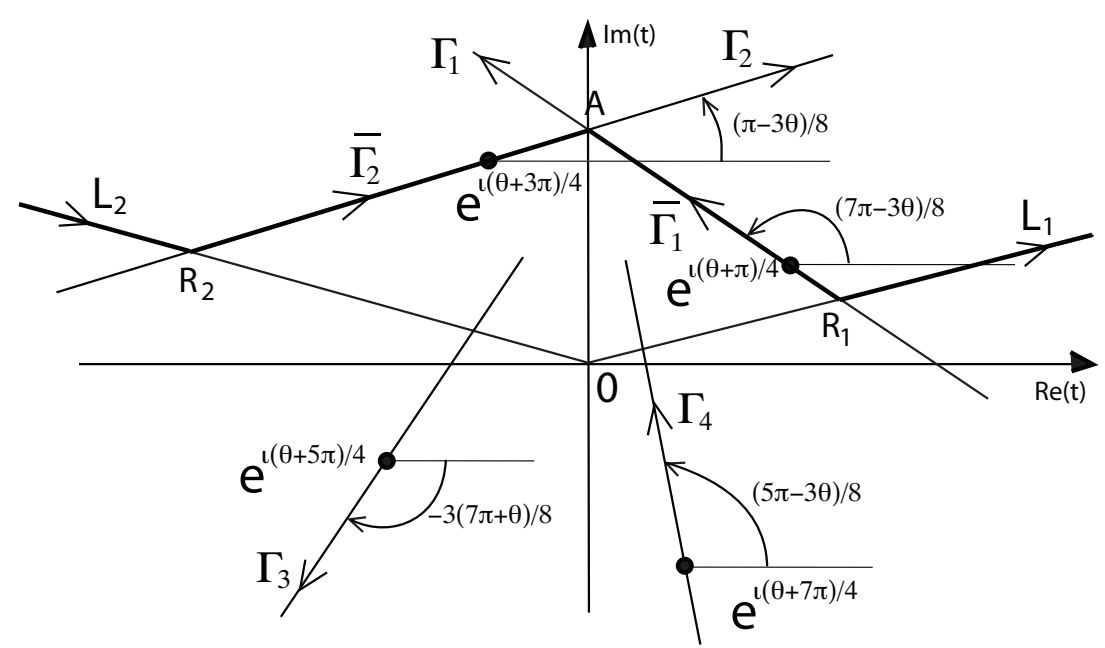

Figure 2: Saddle points $t_{k}:=e^{i(\theta+(2 k-1) \pi) / 4}, k=1,2,3,4$, of the quadratic part $f_{2}(t)$ of the phase function $f(t)$ in (5) and the steepest descent paths $\Gamma_{k}$ of $f_{2}(t)$ at each of the four saddle points. This picture corresponds to $\theta=\pi / 6$. The integration path $L$ in (5) can be deformed to the path $\Gamma=L_{2} \cup \bar{\Gamma}_{2} \cup \bar{\Gamma}_{1} \cup L_{1}$, with $L_{1}:=\left\{r e^{i \pi / 10} ;\left|R_{1}\right|<r<\infty\right\}, L_{2}:=\left\{r e^{9 i \pi / 10} ;\left|R_{2}\right|<r<\infty\right\}$, $\bar{\Gamma}_{1}:=\left[A, R_{1}\right], \bar{\Gamma}_{2}:=\left[R_{2}, A\right], A:=\Gamma_{1} \cap \Gamma_{2}, R_{1}:=\Gamma_{1} \cap L$ and $R_{2}:=\Gamma_{2} \cap L$.

Then, the first point of the asymptotic analysis of (5) is the computation of the steepest descent paths of $f_{2}(t)$. We have that $\phi_{k}=3(\theta+(2 k-3) \pi) / 4, k=1,2,3,4$. Therefore, $\theta_{k}:=-3[(2 k+1) \pi+\theta] / 8, k=1,2,3,4$. Then, the steepest descent paths are the following straights (see Figure 2):

$\Gamma_{k}:=\left\{e^{i(\theta+(2 k-1) \pi) / 4}+r e^{i \theta_{k}} ; \quad \theta_{k}:=-\frac{3}{8}[(2 k+1) \pi+\theta] ;-\infty<r<\infty\right\}, \quad k=1,2,3,4$.

Moreover, at every saddle point $t_{k}, k=1,2,3,4$, we have that the quadratic part of the phase function is

$$
f_{2}(t):=\frac{4}{5} e^{i(5 \theta+(2 k+1) \pi) / 4}+2 i e^{3 i(\theta+(2 k-1) \pi) / 4}\left(t-e^{i(\theta+(2 k-1) \pi) / 4}\right)^{2} .
$$

\section{Deformation of the integration path}

Following [9], the second part of our analysis consists in the deformation of the integration path $L$ in (5) to a new path $\Gamma$ appropriate for the asymptotic analysis: it must contain certain portions $\bar{\Gamma}_{k}$ of some of the four steepest descent straights $\Gamma_{k}, k=1,2,3,4$. In fact, by the use of Cauchy's residue theorem we can deform $L \rightarrow L_{2} \cup \Gamma \cup L_{1}$, where $L_{1}$ and $L_{2}$ are residual portions of the original path $L$ and $\Gamma$ is a portion of one or two appropriate steepest descents $\Gamma_{k}$. Figure 2 shows the four steepest descent paths $\Gamma_{k}$ trough the four saddle points $t_{k}=e^{i(\theta+(2 k-1) \pi) / 4}, k=1,2,3,4$, for the particular case $\theta=\pi / 6$. We have that $L_{1}=\left[R_{1}, \infty e^{i \pi / 10}\right)$ and $L_{2}=\left[R_{2}, \infty e^{9 i \pi / 10}\right)$, where $R_{1}$ and $R_{2}$ are intersections points of $\Gamma_{1}$ and $\Gamma_{2}$ with the original path $L$. (when $\theta=\pi / 6, \Gamma$ is the union of two portions $\bar{\Gamma}_{1}$ and $\bar{\Gamma}_{2}$ of $\Gamma_{1}$ and $\Gamma_{2}$ respectively). 
The precise form of the deformation of the original path $L$ depends on $\theta$ :

$$
\Gamma=\left\{\begin{aligned}
\Gamma_{1} \cup \Gamma_{3} & \text { for }-\pi<\theta<-3 \pi / 5, \\
\Gamma_{1} & \text { for }-3 \pi / 5 \leq \theta<-\pi / 15, \\
\Gamma_{1} \cup \Gamma_{2} & \text { for }-\pi / 5<\theta<\pi / 5, \\
\Gamma_{2} & \text { for } \pi / 15<\theta \leq 3 \pi / 5, \\
\Gamma_{2} \cup \Gamma_{4} & \text { for } 3 \pi / 5<\theta \leq \pi .
\end{aligned}\right.
$$

Figure 3 shows the precise form of the five possible deformations of the path $L$ according to the five regions for the angle $\theta$ detailed above. Therefore, after the deformation,

$$
\Psi(x, y, z)=\left(\frac{|z|}{5}\right)^{1 / 4} \int_{L_{2} \cup \Gamma \cup L_{1}} e^{\left(\frac{|z|^{5}}{5}\right)^{1 / 4} f(t)+i x\left(\frac{|z|}{5}\right)^{3 / 4} t^{3}+i y\left(\frac{|z|}{5}\right)^{1 / 2} t^{2}} d t .
$$

\section{Computation of the integrals over the steepest descent paths}

The third point of our analysis is the approximate computation of the right hand side of (8) when $\Gamma, L_{1}$ and $L_{2}$ are the paths described in the previous section. The following observations are essential in the analysis:

- $\Re[f(t)]$ is a decreasing function in both paths, $L_{1}$ and $L_{2}$, as $|t| \rightarrow \infty$ (t runs from the points $R_{1}$ or $R_{2}$ to the infinity trough $L_{1}$ or $L_{2}$ respectively).

- For $-\pi<\theta \leq \pi$, whatever the path $\Gamma$ is (see (7)), only the saddle points $t_{1}$ and $t_{2}$ are relevant when they are in $\Gamma$ (for any value of $\theta$, at least one of them is in $\Gamma$ ). We have plotted in Figure 4 the functions $\Re\left[f\left(t_{k}(\theta)\right)\right] \chi_{k}(\theta)=\frac{4}{5} \cos [((2 k+1) \pi+5 \theta) / 4] \chi_{k}(\theta)$, $k=1,2,3,4$, for $-\pi<\theta \leq \pi$, where the function $\chi_{k}(\theta)=1$ if the saddle point $t_{k} \in \Gamma$ and $\chi_{k}(\theta)=0$ if $t_{k} \notin \Gamma$ (that is, $\Re\left[f\left(t_{k}(\theta)\right)\right]$ is plotted only for those values of $\theta$ for which $\left.t_{k} \in \Gamma\right)$.

- The difference between the integrals over the whole steepest descent paths $\Gamma_{k}$ and the segments $\bar{\Gamma}_{k}$ that make up $\Gamma$ is exponentially small.

From the above observations we conclude that

$$
\Psi(x, y, z) \sim\left\{\begin{aligned}
\Psi_{2}(x, y, z)-\Psi_{1}(x, y, z) & \text { for }|\theta|<\frac{\pi}{5}, \\
-\Psi_{1}(x, y, z) & \text { for }-\frac{3 \pi}{5} \leq \theta<-\frac{\pi}{15}, \\
\Psi_{3}(x, y, z)-\Psi_{1}(x, y, z) & \text { for }-\pi<\theta<-\frac{3 \pi}{5}, \\
\Psi_{2}(x, y, z) & \text { for } \frac{\pi}{15}<\theta \leq \frac{3 \pi}{5}, \\
\Psi_{2}(x, y, z)+\Psi_{4}(x, y, z) & \text { for } \frac{3 \pi}{5}<\theta \leq \pi,
\end{aligned}\right.
$$




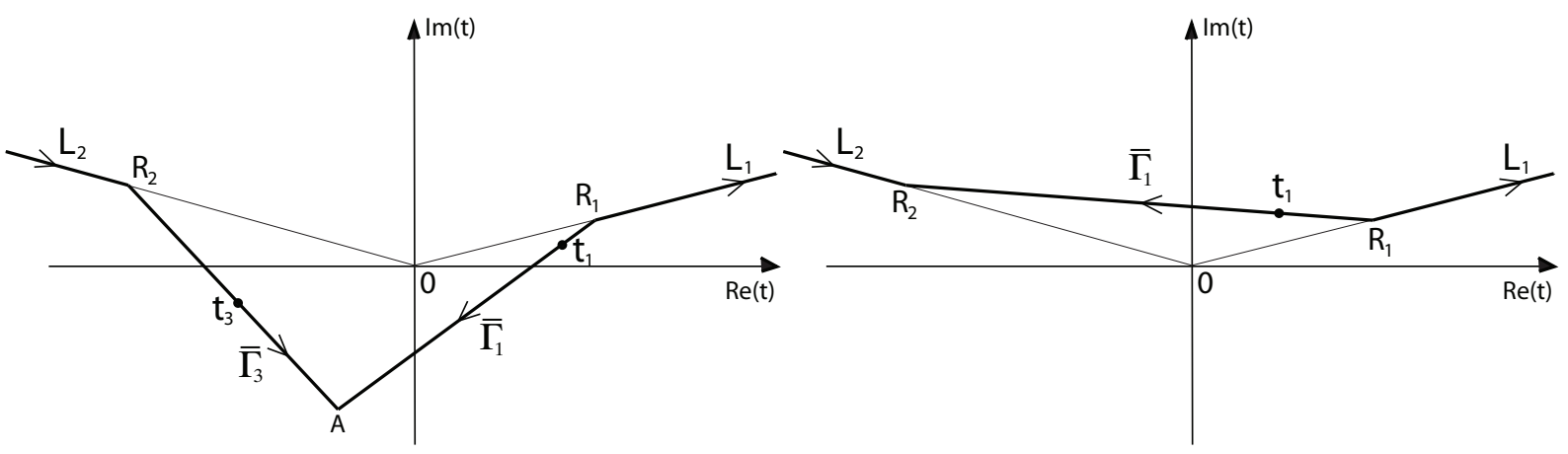

(a)

(b)

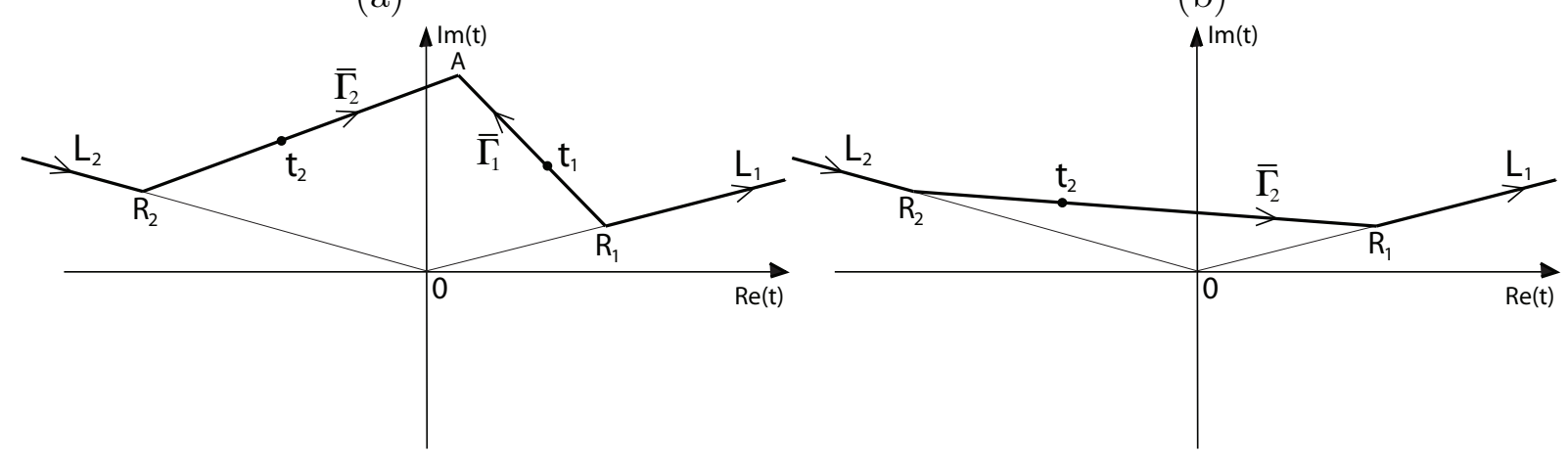

(c)

(d)

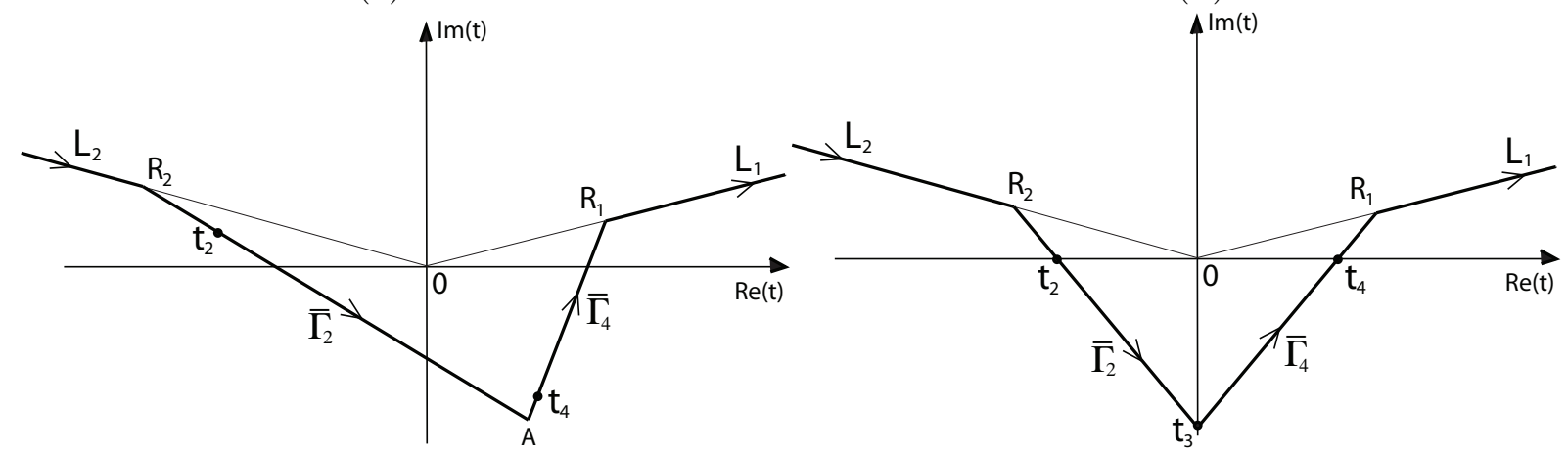

(e)

(f)

Figure 3: Precise form of the deformed path $L_{1} \cup \Gamma \cup L_{2}$ according to the value of $\theta$. In all the figures, $\bar{\Gamma}_{k}$ are pieces of the respective steepest descent paths $\Gamma_{k}, k=1,2,3,4$ : (a) $-\pi<\theta<-3 \pi / 5$ and $\Gamma=\bar{\Gamma}_{1} \cup \bar{\Gamma}_{3}$. (b) $-3 \pi / 5 \leq \theta<-\pi / 15$ and $\Gamma=\bar{\Gamma}_{1}$. (c) $-\pi / 5<\theta<\pi / 5$ and $\Gamma=\bar{\Gamma}_{1} \cup \bar{\Gamma}_{2}$. (d) $\pi / 15<\theta \leq 3 \pi / 5$ and $\Gamma=\bar{\Gamma}_{2}$. (e) $3 \pi / 5<\theta<\pi$ and $\Gamma=\bar{\Gamma}_{2} \cup \bar{\Gamma}_{4}$. (f) $\theta=\pi$ and $\Gamma=\bar{\Gamma}_{2} \cup \bar{\Gamma}_{4}$.

where, for $k=1,2,3,4$,

$$
\Psi_{k}(x, y, z):=\left(\frac{|z|}{5}\right)^{1 / 4} \int_{\bar{\Gamma}_{k}} e^{\left(\frac{|z|^{5}}{5}\right)^{1 / 4} f_{2}(t)+\left(\frac{|z|^{5}}{5}\right)^{1 / 4}\left[f(t)-f_{2}(t)\right]+i x\left(\frac{|z|}{5}\right)^{3 / 4} t^{3}+i y\left(\frac{|z|}{5}\right)^{1 / 2} t^{2}} d t
$$

and $f_{2}(t)$ has been defined in (6). After the change of variables $t \rightarrow u$ given by $t=$ $e^{i((2 k-1) \pi+\theta) / 4}+e^{-3 i[(2 k+1) \pi+\theta] / 8}\left(\frac{5}{|z|^{5}}\right)^{1 / 8} \frac{u}{\sqrt{2}}$ in the integral $(10)$ we find for $k=1,2,3,4$, 


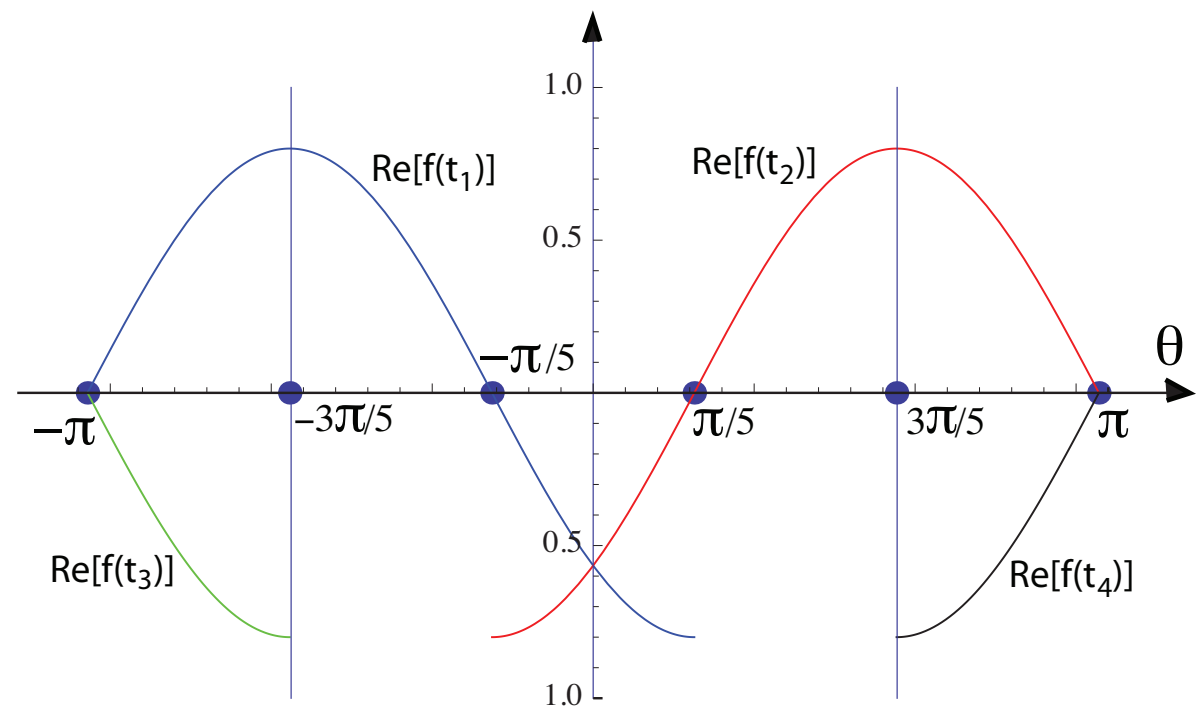

Figure 4: Graphics of the functions $\Re\left[f\left(t_{k}(\theta)\right)\right]=\frac{4}{5} \cos [((2 k+1) \pi+5 \theta) / 4], k=1,2,3,4$, for those values of $\theta$ for which $t_{k} \in \Gamma$.

$$
\Psi_{k}(x, y, z)=\frac{e^{h_{k}(x, y, z)}}{\sqrt{2}\left(5 z^{3}\right)^{1 / 8}} \int_{-a|z|^{5 / 8}}^{b|z|^{5 / 8}} e^{-u^{2}+u w_{k}(x, y, z)} g_{k}(x, y, z, u) d u
$$

where $a$ and $b$ are positive numbers related to the end points $R_{1}, R_{2}$ and $A$ of the piece $\bar{\Gamma}_{k}$ of the saddle straight $\Gamma_{k}$ defining the integral (10). The precise value of $a$ and $b$ is not relevant in the forthcoming analysis. Moreover,

$$
\begin{gathered}
h_{k}(x, y, z):=e^{\frac{i \pi}{4}(6 k-1)} x\left(\frac{z}{5}\right)^{3 / 4}+(-1)^{k} y\left(\frac{z}{5}\right)^{1 / 2}+\frac{4}{5} e^{\frac{1}{4} i \pi(2 k+1)}\left(\frac{z^{5}}{5}\right)^{1 / 4}-\frac{1}{8} i \pi(6 k+3), \\
w_{k}(x, y, z):=\frac{3}{\sqrt{2}} e^{\frac{1}{8} i \pi(2 k-3)} x\left(\frac{z}{5^{5}}\right)^{1 / 8}+\frac{\sqrt{2} e^{-\frac{1}{8} i \pi(2 k+1)} y}{\left(5^{3} z\right)^{1 / 8}} \\
g_{k}(x, y, z, u):=\exp \left\{\frac{a_{k}^{2}(x, y, z)}{z^{5 / 12}} u^{2}+\frac{a_{k}^{3}(x, y, z)}{z^{5 / 8}} u^{3}+\frac{a_{k}^{4}(x, y, z)}{z^{5 / 6}} u^{4}+\frac{a_{k}^{5}(x, y, z)}{z^{25 / 24}} u^{5}\right\}
\end{gathered}
$$

and

$$
\begin{array}{ll}
a_{k}^{2}(x, y, z):=\frac{e^{-\frac{i \pi}{4}(6 k+1)} y}{25^{1 / 4} z^{1 / 3}}-\frac{3 i(-1)^{k} x}{2 \sqrt{5} z^{1 / 12}}, & a_{k}^{3}(x, y, z):=\frac{e^{-\frac{i \pi}{8}(2 k+3)} x}{2 \sqrt{2} 5^{3 / 8} \sqrt{z}}+\frac{5^{1 / 8} \sqrt{2}}{2} e^{\frac{i \pi}{8}(7-10 k)}, \\
a_{k}^{4}(x, y, z):=-\frac{5^{1 / 4} e^{-\frac{i \pi}{4}(2 k+1)}}{4 z^{5 / 12}}, & a_{k}^{5}(x, y, z):=\frac{e^{\frac{5 i \pi}{8}(1-6 k)}}{4 \sqrt{2} 5^{5 / 8} z^{5 / 6}} .
\end{array}
$$


Replacing, for $k=1,2,3,4$, the Taylor expansion of $g_{k}(x, y, z, u)$ at $u=0$ in the integrand of (11), and interchanging sum and integral, we find that, as $|z| \rightarrow \infty$,

$$
\Psi_{k}(x, y, z) \sim \frac{e^{h_{k}(x, y, z)+w_{k}^{2}(x, y, z) / 4}}{\sqrt{2}\left(5 z^{3}\right)^{1 / 8}} \sum_{n=0}^{\infty} \frac{C_{n}^{k}(x, y, z) A_{n}^{k}(x, y, z)}{z^{5 n / 24}},
$$

where, for $k=1,2,3,4$ and $n=0,1,2,3, \ldots, C_{n}^{k}(x, y, z)$ are the Taylor coefficients of $g_{k}(x, y, z, u)$ at $u=0$,

$$
C_{n}^{k}(x, y, z):=\sum_{2 n_{2}+3 n_{3}+4 n_{4}+5 n_{5}=n} \frac{\left[a_{k}^{2}(x, y, z)\right]^{n_{2}}\left[a_{k}^{3}(x, y, z)\right]^{n_{3}}\left[a_{k}^{4}(x, y, z)\right]^{n_{4}}\left[a_{k}^{5}(x, y, z)\right]^{n_{5}}}{n_{2} ! n_{3} ! n_{4} ! n_{5} !}
$$

The function $g_{k}\left(x, y, z, z^{5 / 24} t\right)$ satisfies the differential equation $g_{k}^{\prime}=g_{k} \sum_{m=2}^{5} m a_{k}^{m}(x, y, z) t^{m-1}$ in the variable $t$. From this differential equation it follows that, for $n=1,2,3, \ldots$, the coefficients $C_{n}^{k}(x, y, z)$ may be obtained recurrently in the following form:

$$
C_{n}^{k}(x, y, z)=\frac{1}{n} \sum_{j=2}^{\min \{n, 5\}} k a_{k}^{j}(x, y, z) C_{n-j}^{k}(x, y, z), \quad C_{0}^{k}(x, y, z)=1 .
$$

On the other hand, the functions $A_{n}^{k}(x, y, z)$ are the integrals

$$
\begin{aligned}
A_{n}^{k}(x, y, z):=e^{-w_{k}^{2}(x, y, z) / 4} \int_{-\infty}^{\infty} u^{n} e^{-u^{2}+w_{k}(x, y, z) u} d u \\
=e^{-w_{k}^{2}(x, y, z) / 4}\left\{\begin{array}{cl}
w_{k}(x, y, z) \Gamma\left(\frac{n}{2}+1\right) M\left(\frac{n}{2}+1, \frac{3}{2} ; \frac{w_{k}^{2}(x, y, z)}{4}\right) & \text { if } n \text { odd, } \\
\Gamma\left(\frac{n+1}{2}\right) M\left(\frac{n+1}{2}, \frac{1}{2} ; \frac{w_{k}^{2}(x, y, z)}{4}\right) & \text { if } n \text { even, }
\end{array}\right.
\end{aligned}
$$

where $M(a, b ; z)$ is a confluent hypergeometric function [12]. The first two functions $A_{n}^{k}(x, y, z)$ are

$$
A_{0}^{k}(x, y, z)=\sqrt{\pi}, \quad A_{1}^{k}(x, y, z)=\frac{\sqrt{\pi}}{2} w_{k}(x, y, z)
$$

and, for $n=2,3,4, \ldots$, the remaining ones may be computed from the recurrent relation

$$
A_{n}^{k}(x, y, z)=\frac{w_{k}(x, y, z)}{2} A_{n-1}^{k}(x, y, z)+\frac{n-1}{2} A_{n-2}^{k}(x, y, z) .
$$

This recurrence follows straightforwardly integrating by parts in the integral definition of $A_{n}^{k}(x, y, z)$.

We have that, for $k=1,2,3,4$, the coefficients $C_{n}^{k}$ are $\mathcal{O}(1)$ when $|z| \rightarrow \infty$ and fixed $x$ and $y$. On the other hand, from (14) it is straightforward to see that $A_{n}^{k}(x, y, z)=$ $\mathcal{O}\left(w_{k}(x, y, z)^{n}\right)=\mathcal{O}\left(z^{n / 8}\right)$. Then, apart from the pre-factor in $(12)$, every term inside the series defining the asymptotic expansion of $\Psi_{k}(x, y, z)$ in $(12)$ is of the order $\mathcal{O}\left(z^{-n / 12}\right)$ as $|z| \rightarrow \infty$ for bounded $x$ and $y$. 


\section{Summary of the discussion and numerical experiments}

The asymptotic behavior of the swallowtail integral $\Psi(x, y, z)$ follows from $(9)$ and $(12)$ (see also Figure 4). From (9) and Figure 4 it is clear that the Stokes lines of the swallowtail integral for large $|z| \operatorname{are} \arg (z)= \pm \frac{\pi}{5}$ and $\arg (z)=\pi$, and the complex plane is divided into the three regions depicted in Figure 5, according to the asymptotic behavior of $\Psi(x, y, z)$ :

$$
\begin{aligned}
\Psi(x, y, z) \sim & {\left[\Psi_{2}(x, y, z)-\Psi_{1}(x, y, z)\right] \chi_{(-\pi / 5, \pi / 5)}(\arg z)+\Psi_{2}(x, y, z) \chi_{[\pi / 5,3 \pi / 5]}(\arg z) } \\
+ & {\left[\Psi_{2}(x, y, z)+\Psi_{4}(x, y, z)\right] \chi_{(3 \pi / 5, \pi]}(\arg z)-\Psi_{1}(x, y, z) \chi_{[-3 \pi / 5,-\pi / 5]}(\arg z) } \\
+ & {\left[\Psi_{3}(x, y, z)-\Psi_{1}(x, y, z)\right] \chi_{(-\pi,-3 \pi / 5)}(\arg z) . }
\end{aligned}
$$

where $\chi_{I}(\theta)$ is the characteristic function of the interval $I$ and, for $k=1,2,3,4$, the asymptotic expansions of $\Psi_{k}(x, y, z)$ for large $|z|$ are given in the previous section.

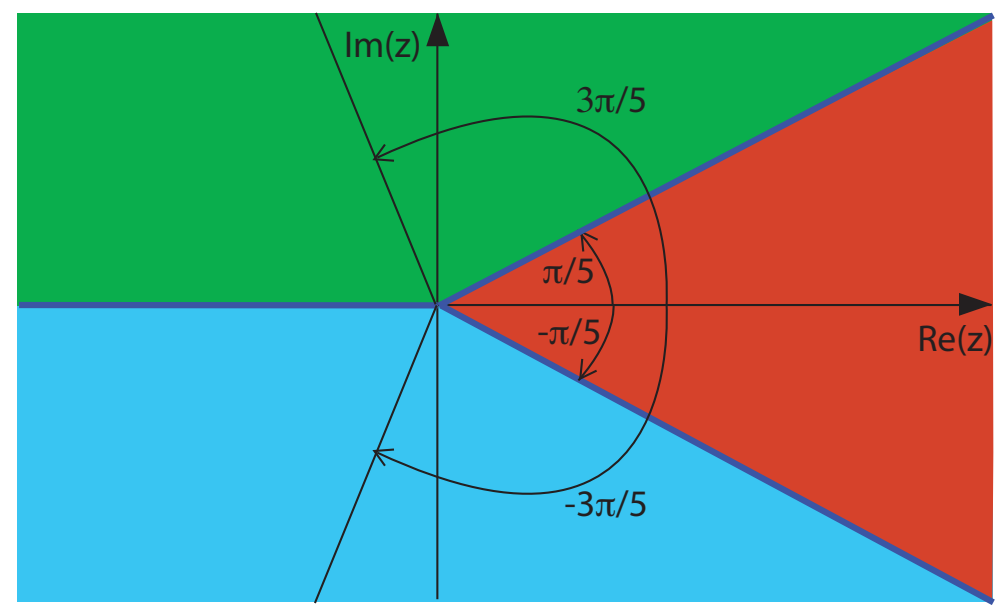

Figure 5: The Stokes lines $\arg (z)= \pm \frac{\pi}{5}$ and $\arg (z)=\pi$ are the thick blue lines depicted in the figure; for large $|z|$, the swallowtail integral behaves differently in the three regions depicted in the figure. The saddle point $t_{1}$ is active for $-\pi<\theta<\pi / 5$. The saddle point $t_{2}$ is active for $-\pi / 5<\theta \leq \pi$. The saddle point $t_{3}$ is active for $-\pi<\theta \leq-3 \pi / 5$. The saddle point $t_{4}$ is active for $3 \pi / 5<\theta \leq \pi$.

The following tables show some numerical experiments about the accuracy of the approximations summarized above. We evaluate the swallowtail integral with the NIntegrate command of Wolfram Mathematica 10.4. All the approximations have been computed by using this software.

\section{Acknowledgments}

Professors Nico M. Temme and Adri Olde Daalhuis are acknowledged by their useful comments and suggestions. The ICMAT of Madrid is acknowledged by the facilities provided 


\begin{tabular}{|c|c|c|c||c|c|c|c|}
\hline \multicolumn{1}{c}{$x=0.5, y=-0.3$} & $z=-0.1, y=0.2 \mathrm{e}^{\mathrm{i} \frac{\pi}{9}}$ \\
\hline$z$ & $n=2$ & $n=4$ & $n=6$ & $z$ & $n=2$ & $n=4$ & $n=6$ \\
\hline $10 \mathrm{e}^{-4 \mathrm{i} \frac{\pi}{5}}$ & 0.065 & 0.038 & $7 . \mathrm{e}-3$ & $10 \mathrm{e}^{\mathrm{i} \pi}$ & 0.037 & 0.025 & 0.0024 \\
\hline $50 \mathrm{e}^{-4 \mathrm{i} \frac{\pi}{5}}$ & 0.029 & $5.3 \mathrm{e}-3$ & $4.7 \mathrm{e}-4$ & $30 \mathrm{e}^{\mathrm{i} \pi}$ & 0.015 & 0.006 & $2.2 \mathrm{e}-4$ \\
\hline $100 \mathrm{e}^{-4 \mathrm{i} \frac{\pi}{5}}$ & 0.02 & $2.3 \mathrm{e}-3$ & $1.4 \mathrm{e}-4$ & $40 \mathrm{e}^{\mathrm{i} \pi}$ & 0.007 & $6.5 \mathrm{e}-4$ & $3 . \mathrm{e}-5$ \\
\hline
\end{tabular}

\begin{tabular}{|c|c|c|c||c|c|c|c|}
\multicolumn{4}{c}{$x=0.4, y=-0.25$} & $x=0.05 \mathrm{i}, y=1.3$ \\
\hline$z$ & $n=2$ & $n=4$ & $n=6$ & $z$ & $n=2$ & $n=4$ & $n=6$ \\
\hline 10 & 0.052 & 0.039 & 0.0028 & $5 \mathrm{e}^{\mathrm{i} \frac{\pi}{16}}$ & 0.12 & 0.08 & 0.007 \\
\hline 25 & 0.045 & 0.008 & 0.0017 & $10 \mathrm{e}^{\mathrm{i} \frac{\pi}{16}}$ & 0.08 & 0.03 & 0.0012 \\
\hline 30 & 0.017 & 0.0077 & $6 . \mathrm{e}-5$ & $20 \mathrm{e}^{\mathrm{i} \frac{\pi}{16}}$ & 0.05 & 0.014 & $2.3 \mathrm{e}-4$ \\
\hline
\end{tabular}

\begin{tabular}{|c|c|c|c||c|c|c|c|}
\hline \multicolumn{1}{c}{$x=-0.5, y=0.1 \mathrm{i}$} & $x=0.2 \mathrm{e}^{\mathrm{i} \frac{\pi}{6}}, y=1.2$ \\
\hline$z$ & $n=2$ & $n=4$ & $n=6$ & $z$ & $n=2$ & $n=4$ & $n=6$ \\
\hline $10 \mathrm{e}^{-2 \mathrm{i} \frac{\pi}{5}}$ & 0.056 & 0.039 & 0.009 & $10 \mathrm{i}$ & 0.06 & 0.037 & 0.002 \\
\hline $40 \mathrm{e}^{-2 \mathrm{i} \frac{\pi}{5}}$ & 0.036 & 0.007 & $7.8 \mathrm{e}-4$ & $100 \mathrm{i}$ & 0.008 & 0.002 & $4.8 \mathrm{e}-5$ \\
\hline $100 \mathrm{e}^{-2 \mathrm{i} \frac{\pi}{5}}$ & 0.024 & 0.002 & $1.6 \mathrm{e}-4$ & $300 \mathrm{i}$ & 0.0024 & $5.6 \mathrm{e}-4$ & $7.4 \mathrm{e}-6$ \\
\hline
\end{tabular}

\begin{tabular}{|c|c|c|c||c|c|c|c|}
\multicolumn{1}{c|}{$x=0.2, y=1.2$} & $x=-4, y=0.5 \mathrm{e}^{\mathrm{i} \frac{\pi}{3}}$ \\
\hline$z$ & $n=2$ & $n=4$ & $n=6$ & $z$ & $n=2$ & $n=4$ & $n=6$ \\
\hline $10 \mathrm{e}^{\mathrm{i}\left(\frac{3 \pi}{5}-0.01\right)}$ & 0.056 & 0.039 & 0.009 & $10 \mathrm{e}^{\mathrm{i}\left(\frac{3 \pi}{5}+0.01\right)}$ & 0.08 & 0.04 & 0.006 \\
\hline $50 \mathrm{e}^{\mathrm{i}\left(\frac{3 \pi}{5}-0.01\right)}$ & 0.018 & 0.005 & $2.3 \mathrm{e}-4$ & $50 \mathrm{e}^{\mathrm{i}\left(\frac{3 \pi}{5}+0.01\right)}$ & 0.03 & 0.005 & $5.5 \mathrm{e}-4$ \\
\hline $100 \mathrm{e}^{\mathrm{i}\left(\frac{3 \pi}{5}-0.01\right)}$ & 0.0095 & 0.002 & $7.7 \mathrm{e}-5$ & $100 \mathrm{e}^{\mathrm{i}\left(\frac{3 \pi}{5}+0.01\right)}$ & 0.02 & 0.0023 & $2.2 \mathrm{e}-4$ \\
\hline
\end{tabular}

\begin{tabular}{|c|c|c|c||c|c|c|c|}
\multicolumn{4}{c}{$x=0.5 \mathrm{i}, y=-0.6$} & $z=-1.3, y=-0.3$ \\
\hline$z$ & $n=2$ & $n=4$ & $n=6$ & $z=2$ & $n=4$ & $n=6$ \\
\hline $10 \mathrm{e}^{\mathrm{i}\left(-\frac{3 \pi}{5}+0.001\right)}$ & 0.1 & 0.04 & 0.0028 & $10 \mathrm{e}^{\mathrm{i}\left(-\frac{3 \pi}{5}-0.001\right)}$ & 0.3 & 0.1 & 0.07 \\
\hline $40 \mathrm{e}^{\mathrm{i}\left(-\frac{3 \pi}{5}+0.001\right)}$ & 0.05 & 0.007 & $5.2 \mathrm{e}-4$ & $70 \mathrm{e}^{\mathrm{i}\left(-\frac{3 \pi}{5}-0.001\right)}$ & 0.1 & 0.019 & 0.004 \\
\hline $100 \mathrm{e}^{\mathrm{i}\left(-\frac{3 \pi}{5}+0.001\right)}$ & 0.027 & 0.002 & $2 . \mathrm{e}-4$ & $130 \mathrm{e}^{\mathrm{i}\left(-\frac{3 \pi}{5}-0.001\right)}$ & 0.08 & 0.01 & 0.002 \\
\hline
\end{tabular}

Table 1: Relative errors in the approximation of the integral $\Psi(x, y, z)$ given in (1) by using (15) with the series truncated after $n$ terms. Several values of $z$ in the regions given in Figure 5 are considered.

during the development of this work. This research was supported by the Ministerio de Economía y Competitividad (MTM2017-83490-P) and the Universidad Pública de Navarra. 


\section{References}

[1] M. V. Berry and C. J. Howls, Integrals with coalescing saddles, in: NIST Handbook of Mathematical Functions, Cambridge University Press, Cambridge, 2010, pp. 775-793 (Chapter 36).

[2] J. N. L. Connor and P. R. Kurtis, A method for the numerical evaluation of the oscillatory integrals associated with the cuspoid catastrophes, J. Phys. A, 15 n. 4 (1982), 1179-1190.

[3] C. Ferreira, J. L. López and E. Pérez Sinusía, The swallowtail integral in the high oscillatory region, Appl. Math. Comput., 339 (2018) 837-845.

[4] C. Ferreira, J. L. López and E. Pérez Sinusía, The swallowtail integral in the high oscillatory region II, to be published in Elec. Trans. Num. Anal.

[5] D. Kaminski, Asymptotic expansion of the Pearcey integral near the caustic, J. SIAM J. Math. Anal., 20 n. 4 (1989), 987-1005.

[6] D. Kaminski, Asymptotics of the swallowtail integral near the cusp of the caustic, J. SIAM J. Math. Anal., 23 n. 1 (1992), 262-285.

[7] J. L. López and P. Pagola, Convergent and asymptotic expansions of the Pearcey integral, J. Math. Anal. Appl., 430 n. 1 (2015) 181-192.

[8] J. L. López and P. Pagola, The Pearcy integral in the highly oscillatory region, Appl. Math. Comput., 275 (2016) 404-410.

[9] J. L. López, P. Pagola and E. Pérez Sinusía, A systematization of the saddle point method. Application to the Airy and Hankel functions, J. Math. Anal. Appl., 354 (2009), 347-359.

[10] Lord Kelvin, Deep water ship-waves, Phil. Mag., 9 (1905), 733-757.

[11] A. B. Olde Daalhuis, On the asymptotics for late coefficients in uniform asymptotic expansions of integrals with coalescing saddles Meth. Appl. Anal., 7 no. 4 (2000), 727-745.

[12] A. B. Olde Daalhuis, Confluent hypergeomeric functions, in: NIST Handbook of Mathematical Functions, Cambridge University Press, Cambridge, 2010, pp. 321-349 (Chapter 13).

[13] R. B. Paris, The asymptotic behaviour of Pearcey's integral for complex variables, Proc. Roy. Soc. London Ser. A., 432 no. 1886 (1991), 391-426.

[14] R. B. Paris and D. Kaminski, Hyperasymptotic evaluation of the Pearcey integral via Hadamard expansions, J. Comput. Appl. Math., 190 (2006), n. 1-2, 437-452.

[15] F. Ursell, Integrals with a large parameter: several nearly coincident saddle points, Proc. Camb. Phil. Soc., 72 (1972), 49-65.

[16] F. Ursell, Ship Hydrodynamics, Water Waves and Asymptotics, Vol. 2 of Collected works of F. Ursell, 1946-1992. Singapore: World Scientific, 1994.

[17] R. Wong, Asymptotic approximations of integrals, Academic Press, New York, 1989. 